\title{
Non Linear Double Diffusive Convection In A Couple Stress Fluid With Rotational Modulation
}

\author{
${ }^{* 1}$ Premila Kollur, ${ }^{2}$ G. Sudhaamsh Mohan Reddy. \\ ${ }^{1}$ Department of Mathematics, Governtment Women's First Grade College, Kalaburgi, India. \\ ${ }^{2}$ Faculty of Science and Technology, ICFAI Foundation for Higher Education, Hyderabad, India . \\ Corresponding author: *Premila Kollur
}

\begin{abstract}
The onset of non linear double diffusive convection in a couple stress fluid with rotation modulation is studied using a truncated representation of Fourier series. The resulting non-autonomous Lorenz model is solved numerically using of Fourier series. The Fehlberg45 method to qualify the heat and mass transport.
\end{abstract}

Keywords: Double diffusive convection,Lorenz Model,Rotational Modulation.Couple Stress Fluid.

Date of acceptance: 31-07-2017

\section{INTRODUCTION.}

The problem of double diffusive convection in porous media has attracted considerable interest during the last few decades because of its wide range of applications, from the solidification of binary mixtures to the migration of solutes in watersaturated soils.Other examples include geophysical systems, electro-chemistry, and the migration of moisture through air contained in fibrous insulation. A comprehensive review of the literature concerning natural convection in fluid-saturated porous media may be found in the books by Ingham and Pop [1, 2], Nield and Bejan [3].

Although the problem of Rayleigh-Benard convection has been extensively investigated for Newtonian fluids, relatively little attention has been devoted to the thermal convection of non-Newtonian fluids. The corresponding problem in the case of a porous medium has also not received much attention until recently. With growing importance of nonNewtonian fluids with suspended particles in modern technology and industries, the investigations of such fluids are desirable. The study of such fluids have applications in a number of processes that occur in industry, such as the extrusion of polymer fluids, solidification of liquid crystals, cooling of metallic plate in a bath, exotic lubrication and colloidal and suspension solutions. In the category of nonNewtonian fluids couple stress fluids have distinct features, such as polar effects. The theory of polar fluids and related theories are models for fluids whose microstructure is mechanically significant. The constitutive equations for couple stress fluids were given by Stokes [4]. The theory proposed by Stokes is the simplest one for micro-fluids, which allows polar effects such as the presence of couple stress, body couple and non-symmetric tensors.

The Rayleigh-Benard situation is often used in rheomatric applications and hence the consideration of the effects of rotational modulation in the control of convection is important from the point of view of applications. In many practical situations, by modulating one of the controlling parameters it is possible to delay or advance the onset of convection. The studies due to Venzian[5],Bhadauria and Khan [6], and pranesh and Sangeetha [7],Malashetty and Padmavati [8] are few examples of problems involving the modulation effect. None of the works, however, quantify the heat and mass transfer. The main objective of this paper is to perform the non-linear stability analysis of double diffusive convection in presence of the modulation of rotational speed in a couple stress fluid using the minimal representation of Fourier series.

\section{MATHEMATICAL FORMULATION}

We consider a viscous, incompressible two component fluid layer of thickness $\mathrm{d}$ and infinite extent in the horizontal direction subject to an adverse temperature gradient and a stabilizing concentration gradient. A Cartesian coordinate system is chosen with the origin in the lower boundary and z-axis vertically upward. In this paper Oberbeck-Boussinesq approximation is assumed to be valid. The sinusoidal applied modulation on the rotation speed is $\omega(t)=\Omega_{0}(1+\varepsilon \cos \omega t) \hat{k}$

Where $\Omega(t)$ represents the Z-component of the rotation speed, $\varepsilon$ is he amplitude of modulation 
and $\omega$ is the frequency of modulation. With the assumptions and approximations frequently made for the study of double diffusive convection in a horizontal two component fluid layer, the basic equations are:

$$
\begin{aligned}
& \nabla \cdot \mathbf{q}=0 \\
& \frac{\rho_{0}}{\varepsilon}\left(\frac{\partial \mathbf{q}}{\partial t}+\frac{1}{\varepsilon} \mathbf{q} \cdot \nabla \mathbf{q}+2 \mathbf{\Omega} \times \mathbf{q}\right)=-\nabla p+\mu \nabla^{2} \mathbf{q}-\mu_{c} \nabla^{4} \mathbf{q}+\rho \mathbf{g},
\end{aligned}
$$

$\frac{\partial T}{\partial t}+(\mathrm{q} \cdot \nabla) T=\kappa_{T} \nabla^{2} T$

$$
\frac{\partial S}{\partial t}+(\mathbf{q} \cdot \nabla) S=\kappa_{S} \nabla^{2} S
$$

$$
\rho=\rho_{0}\left[1-\beta_{T}\left(T-T_{0}\right)\right]+\left[1-\beta_{S}\left(S-S_{0}\right)\right],
$$

where $\mathbf{q}=(u, v, w)$ is the velocity, $T$ is the temperature, $S$ is the solute concentration, $p$ is the pressure, $\rho$ is the density, $T_{0}, S_{0}$ and $\rho_{0}$ are the reference temperature, concentration and density respectively, $\mathrm{g}$ is the acceleration due to gravity, $\mu$ is the fluid viscosity, $\mu^{\prime}$ is the couple stress viscosity, $\beta_{T}$ and $\beta_{S}$ are the thermal expansion coefficient and solute expansion coefficient respectively, $\varepsilon$ is the porosity, $\kappa_{\mathrm{T}}$ and $\kappa_{\mathrm{S}}$ are the thermal diffusivity and solute diffusivity respectively.

\subsection{Basic state}

The basic state of the fluid is quiescent and is given by,

$$
\begin{aligned}
& \mathbf{q}_{\mathrm{b}}=(0,0,0) \quad, \quad p=p_{b}(z) \quad, \quad \rho=\rho_{b}(z), \\
& T=T_{b}(z), S=S_{b}(z) .
\end{aligned}
$$

The temperature $T_{b}(z)$, solute concentration $S_{b}(z)$ , pressure $p_{b}(z)$ and density $\rho_{b}(z)$ satisfy the following equations.

$$
\begin{aligned}
& \frac{\partial p_{b}}{\partial z}=\rho_{b} g, \\
& \frac{d^{2} T_{b}}{d z^{2}}=0, \\
& \frac{d^{2} S_{b}}{d z^{2}}=0,
\end{aligned}
$$

$$
\rho=\rho_{0}\left[1-\beta_{T}\left(T_{b}-T_{0}\right)+\beta_{S}\left(S_{b}-S_{0}\right)\right] .
$$

\subsection{Linear stability analysis}

Let the basic state be perturbed by an infinitesimal thermal perturbation so that

$$
\mathbf{q}=\mathbf{q}_{\mathrm{b}}+\mathbf{q}^{\prime}
$$

$$
\begin{aligned}
& p=p_{b}(z)+p^{\prime}, \rho=\rho_{b}(z)+\rho^{\prime}, \\
& T=T_{b}(z)+T^{\prime}, S=S_{b}(z)+S^{\prime},
\end{aligned}
$$

where primes indicate that the quantities are infinitesimal perturbations. Substituting Eq. (2.11) into Eqs. (2.1)-(2.5) and using basic state equations we obtain the following equations for the perturbed quantities

$\nabla \cdot \mathrm{q}^{\prime}=0$

$\frac{\rho_{0}}{\varepsilon}\left(\frac{\partial q^{\prime}}{\partial t}+\frac{1}{\varepsilon} q^{\prime} \cdot \nabla q^{\prime}+2 \Omega \hat{k} \times q^{\prime}\right)=-\nabla p+\mu \nabla^{2} q^{\prime}-\mu \nabla_{c} \nabla^{4} q^{\prime}+\rho^{\prime} \mathbf{g}$,

$$
\begin{gathered}
\frac{\partial T}{\partial t}+\left(\mathrm{q}^{\prime} \cdot \nabla\right) T+w^{\prime} \frac{\partial T_{b}}{\partial z}=\kappa_{T} \nabla^{2} T \\
\frac{\partial S}{\partial t}+(\mathbf{q} \cdot \nabla) S^{\prime}+w^{\prime} \frac{\partial S_{b}}{\partial z}=\kappa_{S} \nabla^{2} S^{\prime}, \\
\rho^{\prime}=-\rho_{0}\left(\beta_{T} T^{\prime}-\beta_{S} S^{\prime}\right) .
\end{gathered}
$$

We consider only two-dimensional disturbances and define stream function $\psi$ by

$$
\left(u^{\prime}, v^{\prime}\right)=\left(-\frac{\partial \psi}{\partial z}, \frac{\partial \psi}{\partial x}\right)
$$

which satisfy the continuity Eq. (2.12).

$$
\text { Eliminate pressure from Eq. (2.13) }
$$
introducing the stream function $\psi$ and nondimesionalizing the resulting equation, as well as Eqs. (2.14) and (2.15) using the following nondimensional parameters

$$
\begin{aligned}
& \left(x^{*}, z^{*}\right)=\left(\frac{x}{d}, \frac{z}{d}\right), t^{*}=t\left(d^{2} / \kappa_{T}\right), \psi^{*}=\psi / \kappa_{T}, \\
& T^{*}=T / \Delta T, S^{*}=S / \Delta S,
\end{aligned}
$$

we obtain 


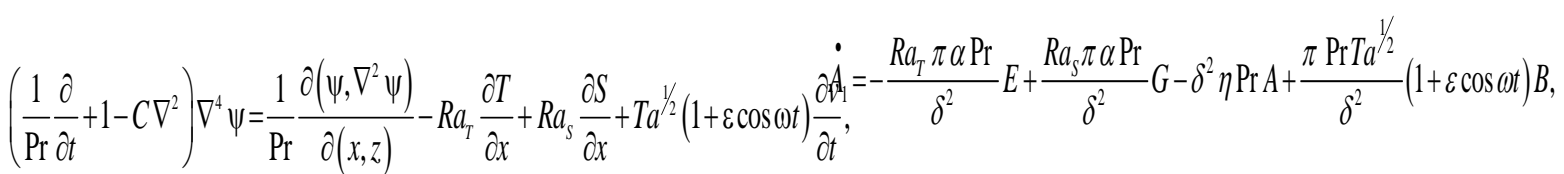

$$
\begin{aligned}
& \frac{\partial T}{\partial t}=-\frac{\partial \psi}{\partial x}+\frac{\partial(\psi, T)}{\partial(x, z)}+\nabla^{2} T \\
& \frac{\partial S}{\partial t}=-\frac{\partial \psi}{\partial x}+\frac{\partial(\psi, S)}{\partial(x, z)}+\tau \nabla^{2} S
\end{aligned}
$$

The asterisks have been dropped for simplicity Eqs. (2.19)-(2.21) are solved stress-free, isothermal, vanishing couple stress boundary conditions namely

$$
\psi=\frac{\partial^{2} \psi}{\partial z^{2}}=T=S=0 \text { at } z=0,1
$$

\section{NON-LINEAR THEORY}

In this section we consider the nonlinear analysis using a truncated representation of Fourier series considering only two terms. We consider the early stages of nonlinear convection, when the basic structure of the convection is still determined by the behavior of the linearized solution. In the immediate vicinity of the stability boundary, we could develop a weakly nonlinear analysis.

We will assume that, close to the threshold of convection, the basic circulation remains undisturbed but the temperature and concentration fields are distorted by the addition of a second harmonic with no $\mathrm{x}$-dependence, so that

$\psi=A(t) \sin (\pi a x) \sin (\pi z)$

$V=B(t) \sin (\pi a x) \cos (\pi z)+D(t) \sin (2 \pi x)$

$T=E(t) \cos (\pi a x) \sin (\pi z)+F(t) \sin (2 \pi z)$,

$S=G(t) \cos (\pi a x) \sin (\pi z)+H(t) \sin (2 \pi z)$,

where

the

amplitudes

$A(t), B(t), D(t), E(t), F(t), G(t)$ and $H(t)$

are to be determined from the dynamics of the system.

Substituting Eqs. (3.1)-(3.4) into Eqs. (2.19)-(2.21) and equating the coefficients of like terms we obtain the following non-linear autonomous system of differential equations

$$
\dot{B}_{1}=-2 \pi^{2} \alpha A D-\delta^{2} \operatorname{Pr} \eta B-A \pi \operatorname{Pr} T a^{1 / 2}(1+\varepsilon \cos \omega t),
$$

$$
\dot{B}_{2}=-4 \pi^{2} \alpha^{2} \operatorname{Pr} D-\frac{\pi^{2} \alpha}{2} A B-16 C \pi^{4} \alpha^{4} \operatorname{Pr} D,
$$

$\dot{E}_{1}=-\pi \alpha A-\delta^{2} \tau G-\pi^{2} \alpha A E$

$\dot{E}_{2}=-4 \pi^{2} \tau F+\frac{\pi^{2} \alpha}{2} A E$

where the over dot denotes the time derivative.

The generalized Lorenz [18] model (3.5)(3.9) is uniformly bounded in time and possesses many properties of the full problem. Also the phase space must contract, In order to prove volume contraction, we must show that velocity field has a constant negative divergence, Indeed,

$\frac{\partial \dot{A}_{1}}{\partial A_{1}}+\frac{\partial \dot{B}_{1}}{\partial B_{1}}+\frac{\partial \dot{B}_{2}}{\partial B_{2}}+\frac{\partial \dot{E}_{1}}{\partial E_{1}}+\frac{\partial \dot{E}_{2}}{\partial E_{2}}=-\left[2 \delta^{2} \eta \operatorname{Pr}+16 C \pi^{4} \alpha^{4} \operatorname{Pr}+4 \pi^{2}\left(1+\tau^{2}\right)\right]$

which is always negative and therefore the system is bounded and dissipative. As a result, the trajectories are attracted to a set of measure zero in the phase space: in particular they may be attracted to a fixed point, a limit cycle or, perhaps, a strange attractor.

\section{Heat and mass transports}

In the study of convection in fluids, the quantification of heat and mass transport is important. This is because the onset of convection, as Rayleigh number is increased, is more readily detected by its effect on the heat and mass transport. In the basic state, heat and mass transport is by conduction alone.

If $H$ and $J$ are the rate of heat and mass transport per unit area respectively, then

$$
\begin{aligned}
& H=-\kappa\left\langle\frac{\partial T_{\text {total }}}{\partial z}\right\rangle_{z=0}, \\
& J=-D\left\langle\frac{\partial S_{\text {total }}}{\partial z}\right\rangle_{z=0},
\end{aligned}
$$


where the angular bracket corresponding to a horizontal average and

$$
\begin{aligned}
& T_{\text {total }}=T_{0}-\Delta T \frac{z}{d}+T(x, z, t), \\
& S_{\text {total }}=S_{0}-\Delta S \frac{z}{d}+S(x, z, t) .
\end{aligned}
$$

The thermal and shear wood numbers are defined by

$$
\begin{aligned}
& N u=\frac{H}{\kappa \Delta T / d}=(1-2 \pi F), \\
& S h=\frac{J}{D \Delta T / d}=(1-2 \pi G) .
\end{aligned}
$$

The amplitude $F(t)$ and $G(t)$ determined from the dynamics of the generalized Lorenz system (3.1)(3.4) which can obtained by solving the system using the Runge -Kutta-Fehlberg45 method that uses an adaptive step-size.

\section{RESULTS AND DISCUSSION}

In the present paper, the non-linear stability analysis of double diffusive convection with rotational modulation in a couple stress fluid is studied. In the case when modulation is present the critical Rayliegh number for onset of convection is larger than the one predicted by the onset of convection without modulation. The Lorenz model obtained in the problem is solved using Runga-KuttaFehliberg45 method with adaptive step size. The initial condition that we have used for numerical integration of equations (3.1)-(3.4) are:

$\mathrm{A}(0)=\mathrm{B}(0)=\mathrm{D}(0)=\mathrm{E}(0)=\mathrm{F}(0)=\mathrm{G}(0)=\mathrm{H}(0)=2$.

It is observed that Taylor number and amplitude of modulation lead to a reduced heat and mass transfer situation compared to that of the case of non-modulation problem. The couple stress parameter represents the presence of suspended particles in the fluid and hence this parameter only adds to the effects of Taylor number. Ta, and amplitude of modulation, $\epsilon$, on the Nusselt and Sherwood number. The three parameters ensure that the system is stable leading to a situation where in rheometric measurements are possible.

\section{Nomenclature}

$A_{m n} \quad$ amplitude of streamline perturbation

$B_{m n} \quad$ amplitude of thermal perturbation

$E_{m n} \quad$ amplitude of concentration perturbation
C couple stress parameter, $\frac{\mu^{\prime}}{\mu d^{2}}$

$d \quad$ height of the fluid layer

Da Darcy number, $\frac{k}{d^{2}}$

$g$ gravitational acceleration, $(0,0,-g)$

$\mathrm{Nu} \quad$ Nusselt number

Sh Sherwood number

$k \quad$ permeability

$l, m \quad$ wave numbers in the horizontal plane

$p \quad$ pressure

Pr Prandtl number, $\frac{\mathrm{v}}{\kappa_{T}}$

q velocity vector, $(u, v, w)$

$R a_{T} \quad$ thermal Rayleigh number, $\frac{\beta_{T} g \Delta T d k}{v \kappa_{T}}$

$R a_{s} \quad$ solute Rayleigh number, $\frac{\beta_{s} g \Delta S d k}{v \kappa_{s}}$

$S \quad$ solute concentration

$\Delta S \quad$ salinity difference between the plates

$T$ temperature

$\Delta T \quad$ temperature difference between the plates

$t \quad$ time

$x, y, z \quad$ space coordinates

\section{Greeks symbols}

$\alpha \quad$ wavenumber

$\beta_{s} \quad$ solute expansion coefficient

$\beta_{T} \quad$ thermal expansion coefficient

$\delta \quad \pi^{2}\left(\alpha^{2}+1\right)$

$\phi \quad$ dimensionless concentration

$\eta \quad 1+C \delta^{2}$

$\kappa \quad$ thermal diffusivity

$\mu \quad$ dynamic viscosity

$\mu_{c} \quad$ couple stress viscosity

$v \quad$ kinematic viscosity, $\mu / \rho_{0}$

$\theta \quad$ dimensionless temperature

$\rho$ density

$\sigma \quad$ growth rate

$\tau \quad$ diffusivity ratio, $\kappa_{\mathrm{S}} / \kappa_{\mathrm{T}}$

$\omega$ frequency

$\psi \quad$ stream function

\section{Subscript}

$b \quad$ basic state

c critical

0 reference value 
Superscript

* dimensionless quantity

perturbed quantity

\section{REFERENCES}

[1]. Ingham D.B. and Pop I. (eds.), Transport Phenomena in Porous Media,

[2]. Pergamon, Oxford, 1998.

[3]. Ingham D.B. and Pop I. (eds.), Transport Phenomena in Porous Media, Volume III, Elsevier, Oxford, 2005.

[4]. Nield, D.A. and Bejan, A., Convection in porous media, $\left(3^{\text {nd }}\right.$ edn) Springer-Verlag, New York (2006).

[5]. V.K. Stokes, Couple stresses in fluids, Phys. Fluids, 9 (1966) 1709-1716.

[6]. Venezian,G.effect of modulation on the onset of thermal convection,J.Fluid Mech 35(2),(1969).243.

[7]. Bhadauria,B.S and Khan,A Modulated centrifugal convection in a vertical layer porous distant from the axix of rotation.Transp.Porous Med.,79,(2009),255.

[8]. Pranesh, S and Sangeetha George. Effect of magnetic field on the onset of Rayleigh Benard convection in Boussinessq stokes suspensions with time periodic boundary temperatures. IJAMM,(2010), 6(6), 38.

[9]. M. S. Malashetty and D. Basavaraja, Effect of thermal / gravity modulation on the onset of Rayleigh -Benard convection in a couple stress fluid, Int. J. Trans. Phenomena, 7(2005)31-44.

\footnotetext{
International Journal of Engineering Research and Applications (IJERA) is UGC approved Journal with S1. No. 4525, Journal no. 47088. Indexed in Cross Ref, Index Copernicus (ICV 80.82), NASA, Ads, Researcher Id Thomson Reuters, DOAJ.

Premila Kollur. "Non Linear Double Diffusive Convection In A Couple Stress Fluid With Rotational Modulation." International Journal of Engineering Research and Applications (IJERA) 7.7 (2017): 25-29.
} 